\title{
Quadruped Robot Leg Optimization Based on a Multi-Objective Genetic Algorithm
}

\author{
Yongnian ZHANG*, Xinsheng WANG*, Yuhong XIN**, Yang WU*, Min KANG*, \\ Xiaochan WANG* \\ *College of Engineering, Nanjing Agricultural University, Nanjing, 210031, China, \\ E-mail: wangxiaochan@njau.edu.cn (corresponding author) \\ **College of Mechanical and Electrical Engineering, Jinling Institute of Technology, Nanjing, 211169, China, \\ cross'ref http://dx.doi.org/10.5755/j01.mech.23.6.19854
}

\section{Introduction}

The velocity of quadruped robots has speed up with the change from the walk gait to the trot gait [1], and is currently moving in the direction of high-speed development.

In nature, the realization of high-speed movement in animals is mainly by a high stride frequency and low duty factor [2]. In the swing phase, the legs stretch rapidly and switch their orientations at the start and end moments, which requires an extremely high acceleration. This results in a high ground reaction force in the static phase. When a human runs at $4.5 \mathrm{~m} / \mathrm{s}$, the typical maximum normal ground reaction force on each leg is approximately three times the bodyweight [3], and when a dog gallops at $9 \mathrm{~m} / \mathrm{s}$, the corresponding force is 2.6 times [4].

The leg mechanism is viewed as a key component of quadruped robots, and its DOF, configuration and weight have great influences on the motion performance [5]. To satisfy the requirements of high acceleration and a large reaction force, the following methods are generally adopted.

1. Enhance the drive capacity. The most typical case is the robot cheetah [6] developed by the MIT Bionic Robotics Laboratory, which can leap over 0.4 meters high at $2.4 \mathrm{~m} / \mathrm{s}$. Its superior performance is mainly due to the self-developed high-performance brushless DC torque motor with high power density (the peaking torque of a $1-\mathrm{kg}$ motor can reach $60 \mathrm{Nm}$ [7]). The use of these motors as a power source will provide the legs with a fairly high acceleration.

2. Minimize the inertia of the legs. All of the drive motors are set up near the shoulder, with one motor driving each shoulder joint and another driving each knee joint through a linkage mechanism. This will effectively diminish the rotational inertia of the legs. Both the KOLT robot [8] from Stanford University and the Cheetah robot [9] the Swiss Federal Institute of Technology use rope, while the StarlETH robot [10] developed by the Swiss Federal Institute of Technology uses a chain transmission mechanism to drive the knee joint. Meanwhile, decreasing the weight [11] of the legs on the premise of ensuring the leg strength can also reduce the inertia.

3 . Introduce compliance. Both a spring and elastic buffer device are used in the legs, such as BigDog [12], the Cheetah robot [13] developed by Boston Dynamics, the Cheetah robot [9] from the Swiss Federal Institute of Technology and the MIT Cheetah [6].
4. Another common design technique to speed up the robot velocity is using high strength to weight ratio materials such as carbon fibre [14],steel, aluminium alloys [15], magnesium alloy [16] and titanium [17].Using tububots $[12,14,18]$.

When constructing the prototype, measures 2,3 and 4 can easily be accomplished. However, the high acceleration of the legs necessitates the use of a more powerful motor, which will lead to heavier legs. This places a high technical threshold on the development of the torque motor, which will expend considerable time and funds.

This paper introduces a novel electric drive robot leg based on the double four-bar mechanism, which is used to transform the continuous rotation of motors into the back and forth motion of a leg. This scheme avoids the frequent shifts between the acceleration and deceleration of the motors, thus enabling a higher rotation speed. However, the link dimensions will have a significant impact on the dynamic characteristics and endurance performance of the robot, so it is vital to find a reasonable set of link dimensions.

Based on a full analysis of the kinematic and dynamic characteristics of the double four-bar mechanism, the mathematical relationships between the peak torque, peak angular velocity, total energy consumption in a single gait cycle and the link dimensions are set up, and then a multi-objective optimization model is constructed, which adopts the gamultiobj algorithm to obtain the Pareto optimal solution set and the maximizing deviation method to determine the weight of each target. In this way, the best link parameters satisfying the requirements from the Pareto optimal solution are obtained, thereby providing theoretical guidance for the design of the double four-bar mechanism of the quadruped robot.

\section{Structural design of the leg}

To reduce the inertia of the legs and improve the dynamic characteristics of the robot [19], we need to use lightweight materials and adopt measures to reduce the weights and we should also arrange the drive motors on the robot body in the optimal fashion. This paper introduces a novel electric drive robot leg based on the double four-bar mechanism, as shown in Fig. 1. The positions fixing the hip and knee motors by bolting to the robot body are noted as $A$ and $D$, respectively. $D H, H I$, and $I K$ are the humerus, radius and foot, respectively, and a parallel four-bar linkage GHIJ is adopted to guarantee that the humerus and foot are kept parallel and increase the leg stiffness. $A B C D$ 
constitutes a crank rocker mechanism, and DEFG constitutes a double-rocker mechanism. These two four-bar linkages drive the movement of the robot humerus and radius.

$A B C D$ : Compared with the knee motor, the hip motor needs to bear a greater load, and the frequent shifting between acceleration and deceleration will have a greater impact. Therefore, a crank rocker mechanism is used to transform the continuous rotation of the motors to the back and forth motion of the leg, where crank $A B$ driven by the hip motor rotates around point $A$, and linkage $B C$ transmits the power to rocker $C D$. Because $D C G H$ is a rigid body, the robot humerus can quickly swing back and forth.

$D E F G$ : Rocker $D E$ driven by the knee motor swings back and forth, and the motion is transmitted to the other rocker $G F$ through linkage $E F$, where $G F J$ can be considered a rigid body. Then, the robot radius $H I$ can swing quickly through the parallel four-bar linkage GHIJ.

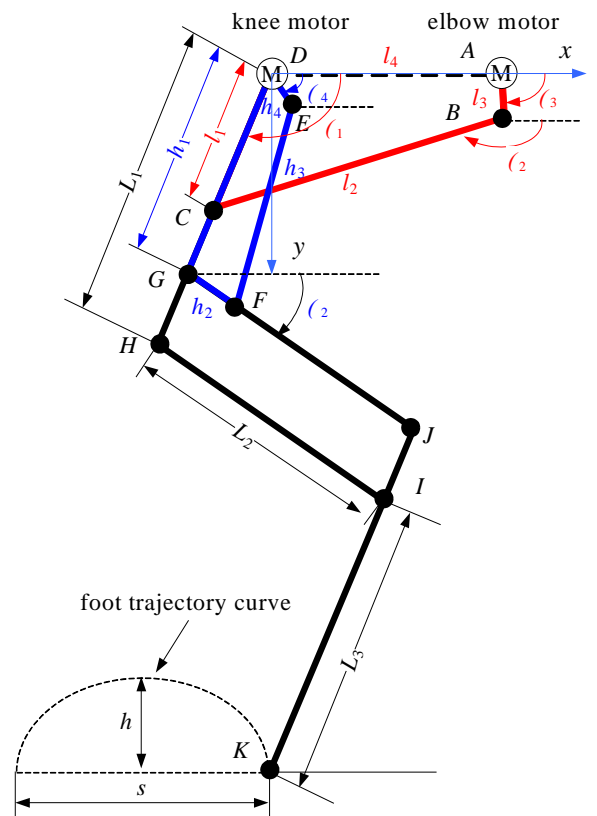

Fig. 1 Designed robot leg based on the double four-bar mechanism

For the robot with three-segment legs $\left(L_{1} 、 L_{2} 、 L_{3}\right.$ ) shown in Fig. 1, there exist a few recent studies on the relative segment lengths of three-segment legs and their influence on the energy efficiency and achievable acceleration. In terms of energy, a relative ratio among the humerus, radius and foot lengths of 0.39:0.45:0.16 [20] seems to be optimal. However, in nature, this is only seen for humans and large quadruped animals and comes at the cost of a rather low acceleration. For smaller mammals, an equal segment length ratio of 0.33:0.33:0.33 [21] seems to be optimal for locomotion. The advantage of this configuration is a large working range and good acceleration properties. Therefore, this equal segment length ratio is adopted in this paper.

\section{Kinematic and dynamic analysis}

The kinematic equation must be established to achieve motion control of the quadruped robot. As shown in Fig. 1, an $x-y$ coordinate system is established, with the origin located at shoulder joint $D$ and the $x$ axis along the frame direction.

Considering the linkage of $D H, H I$ and $I K$, the D$\mathrm{H}$ coordinate system can be determined. Given the foot trajectory point $K$, the angles of the hip motor $\theta_{1}$ and knee motor $\alpha_{2}$ can be calculated by inverse kinematics.

$$
\left\{\begin{array}{l}
\theta_{1}=\theta_{K}+\arccos \frac{L_{K}^{2}+\left(L_{1}+L_{3}\right)^{2}-L_{2}^{2}}{2\left(L_{1}+L_{3}\right) L_{K}} \\
\alpha_{2}=\theta_{K}-\arccos \frac{L_{K}^{2}+L_{2}^{2}-\left(L_{1}+L_{3}\right)^{2}}{2 L_{2} L_{K}}
\end{array},\right.
$$

where $\theta_{K}=\arctan \left(x_{K}, y_{K}\right), L_{K}=\sqrt{x_{K}^{2}+y_{K}^{2}}$.

To guarantee the walking stability and small undulation of the body, the foot trajectories should be smooth with a low contact impact. Thereby, the low contact impact gait planning method proposed by Wang Lipeng et al. [22], with particular attention paid to the foot motion trajectory, is selected. The trot gait is adopted due to its advantages of high velocity and good stability. The motion parameters are shown in Table 1.

Table 1

Motion parameters adopted in this paper

\begin{tabular}{|c|c|}
\hline Parameter & Value \\
\hline Step length $s$ & $300 \mathrm{~mm}$ \\
\hline Step height $h$ & $70 \mathrm{~mm}$ \\
\hline Period $T$ & $0.6 \mathrm{~s}$ \\
\hline
\end{tabular}

Substituting the motion parameters into Eq. (1), we can easily obtain the range of shoulder joint angles $\theta_{1} \in\left[88.36^{\circ}, 137.62^{\circ}\right]$ shown in Fig. 2.

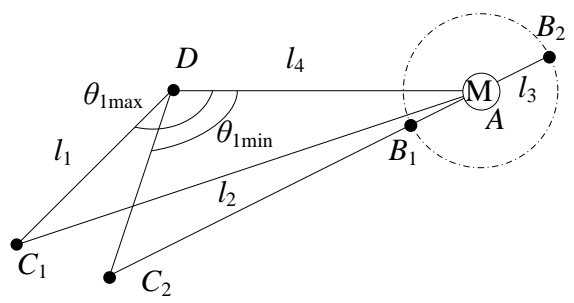

Fig. 2 Limiting positions of crank rocker mechanism

In Fig. 2, $A B_{1} C_{1} D$ and $A B_{2} C_{2} D$ are the two limiting positions of the crank rocker mechanism. The dimensions of linkages $B C$ and $A B$ can be obtained according to the geometrical relationship:

$$
\left\{\begin{array}{l}
l_{2}=(a+b) / 2 \\
l_{3}=(a-b) / 2
\end{array},\right.
$$

where

$$
a=\sqrt{l_{1}^{2}+l_{4}^{2}-2 l_{1} l_{4} \cos \theta_{1 \max }}, b=\sqrt{l_{1}^{2}+l_{4}^{2}-2 l_{1} l_{4} \cos \theta_{1 \min }} .
$$


Set up the vector loop equation of $A B C D$ and project it through the $x$ and $y$ directions as follows.

$$
\left\{\begin{array}{l}
l_{1} \cos \theta_{1}+l_{2} \cos \theta_{2}=l_{4}+l_{3} \cos \theta_{3}, \\
l_{1} \sin \theta_{1}+l_{2} \sin \theta_{2}=l_{3} \sin \theta_{3} .
\end{array}\right.
$$

The hip motor angle $\theta_{3}$ can be quantified by solving the simultaneous equations (1) (3). In the same way, the knee motor angle $\alpha_{4}$ can be obtained according to the knee joint angle $\alpha_{2}$ in the double-rocker mechanism $D E F G$.

To uniquely determine and easily measure the states of the leg, the variables of the constraint joints $q=\left[\theta_{3}, \alpha_{4}\right]^{\mathrm{T}}$ are used for describing the motion, and then the corresponding generalized driving force $F$ can be obtained by Lagrange's equation:

$$
F_{i}=\frac{d}{d t}\left(\frac{\partial E_{k}}{\partial \dot{q}_{i}}\right)-\frac{\partial E_{k}}{\partial q_{i}}+\frac{\partial E_{p}}{\partial q_{i}}(i=1,2),
$$

where $E_{k}, E_{p}$, and $q_{i}$ are the total kinetic energy, total potential energy and generalized coordinates of the system, respectively.

Suppose that the foot trajectory is $P_{K}$, the gravitational acceleration vector is $g$, and the CM (center of mass) vector, CV (center of velocity) vector, angular velocity vector, moment of inertia and the mass of each linkage are $P_{i}, v_{i}, \omega_{i}, I_{i}$ and $m_{i}$, respectively. Each linkage is simplified to a homogeneous bar, and then the kinetic energy $E_{k}$ is defined as the sum of each linkage's kinetic energy, which is:

$$
E_{k}=0.5 \sum_{i=1}^{n}\left(m_{i} v_{i}^{\mathrm{T}} v_{i}+\omega_{i}^{\mathrm{T}} I_{i} \omega_{i}\right)
$$

Let $N$ represent the ground reaction force and $M$ represent the mass of the robot. Considering that a quadruped robot has two feet on the ground at the same time while walking with a trot gait, the ground reaction force of each leg is considered to approximate a fixed magnitude $N=-\eta M g(\eta=0.5 \sim 2)$ in the static phase and 0 in the swing phase. Therefore, the ground reaction force can be processed similarly to the gravitational potential energy, and the total potential energy can be expressed as:

$$
E_{p}=\sum_{i=1}^{n}\left(-m_{i} g P_{i}-N P_{G}\right)
$$

The corresponding joint torque can be determined by solving equations $(4) \sim(6)$ simultaneously as:

$$
\tau=D(q) \ddot{q}+H(q, \dot{q}) \dot{q}+G(q),
$$

where $D(q), H(q, \dot{q})$, and $G(q)$ are the inertia matrix, centrifugal and Coriolis force vector and gravity vector of the leg, respectively.
Suppose that the gait period is $T$ and the total energy consumption of each leg in a gait period can be expressed as:

$$
E=\int_{0}^{T} \tau \dot{q} d t
$$

\section{Establishment and analysis of multi-objective optimization model}

As with the single-objective optimization issue, the establishment of the multi-objective optimization model includes objective functions, decision variables and constraints [23]. Its mathematical model can be expressed as follows:

$$
\left\{\begin{array}{llc}
\min & f_{m}\left(x_{1}, x_{2}, \cdots, x_{n}\right) \quad(m=1,2, \cdots, M), \\
\text { s.t. } & g_{k}\left(x_{1}, x_{2}, \cdots, x_{n}\right) \geq 0 & (k=1,2, \cdots, K), \\
& h_{l}\left(x_{1}, x_{2}, \cdots, x_{n}\right)=0 & (l=1,2, \cdots, L), \\
& x_{i}^{L} \leq x_{i} \leq x_{i}^{U} & (i=1,2, \cdots, n) .
\end{array} .\right.
$$

In Eq. (9), $X \subset R^{n}$ is the decision space, $x=\left(x_{1}, x_{2}, \cdots, x_{n}\right) \in X . x_{i}^{L}$ and $x_{i}^{U}$ are the upper and lower bounds of variable $x_{i}$.

$\Omega=\left\{x \mid g_{k}(x) \geq 0, h_{l}(x)=0, x_{i}^{L} \leq x_{i} \leq x_{i}^{U}\right\}$ is the feasible set of the multi-objective problem, and clearly $\Omega \subseteq X$.

\subsection{Decision variables}

For the three-segment leg shown in Fig. 1, the dimensions of $A B C D$ and $D E F G$ need to be optimized. First, the installation distance between the two motors can be determined $l_{4}=130 \mathrm{~mm}$ with reference to the common size of the servo motors on the market. Then, the frame length of $D G h_{1}=110 \mathrm{~mm}$ is selected to avoid movement interference of the parallel four-bar linkages. Considering that the four-bar linkages should be as compact and small as possible, the dimension of the rocker $D E$ is determined as $h_{4}=20 \mathrm{~mm}$. Therefore, the linkages that need to be optimized include $l_{1}, h_{2}, h_{3}$, which means that the decision variables of such a multi-objective optimization model are $x=\left(l_{1}, h_{2}, h_{3}\right)$.

\subsection{Constraints}

1. Boundary constraints.

Consider the possible scope of the dimensions, and estimate the range of the optimal solutions to establish the boundary conditions:

$$
\left\{\begin{array}{l}
h_{\text {min }} \leq h_{1} \leq h_{\max }, \\
l_{\text {min }} \leq l_{i} \leq l_{\max } \quad i=2,3 .
\end{array}\right.
$$

2. Geometric constraints.

The movable sufficient condition of the four-bar linkage mechanisms is derived on the basis of the assembly conditions, which are: 


$$
\left|l_{1}-l_{2}\right| \leq l_{B_{1} D} \leq l_{1}+l_{2} .
$$

\section{Dynamic constraints.}

To guarantee the smooth movement and high transmission efficiency of the four-bar linkage, the minimum transmission angle $\gamma_{\text {min }}$ should be not less than the allowable transmission angle $\gamma$ :

$$
\gamma_{\min } \geq[\gamma]
$$

\subsection{Objective functions}

\section{Torque performance.}

The output torque of the servo motor is an important factor that has some effect on the dynamic performance of the quadruped robot. Motor selection for robot design should guarantee that the peak torque of the motor is greater than the load torque, so reducing the peak load torque makes it possible to choose a lower power motor with a light weight and thereby improve the walking performance, load capacity and endurance performance. Thus, the goal of optimization is to reduce the load torque of the joint motors as much as possible.

$$
\left\{\begin{array}{l}
\min f_{1}(x)=\min \left(\tau_{1 \max }\right), \\
\min f_{2}(x)=\min \left(\tau_{2 \max }\right) .
\end{array} .\right.
$$

\section{Speed performance.}

The maximum speed of the hip and knee joint motor is also an important basis for the motor selection. At present, the common servo motor cannot achieve high speed and high torque simultaneously, so we should make a good trade-off between the speed and torque. In general, the angular velocity of the motor should be as small as possible in a single gait period. This can not only reduce the performance requirement for the motor but also avoid the distortion of control caused by an over-high angular velocity to which the servo system cannot respond to in time. Then, the objective functions of the speed performance can be listed as follows:

$$
\left\{\begin{array}{l}
\min f_{3}(x)=\min \left(\dot{q}_{1 \max }\right), \\
\min f_{4}(x)=\min \left(\dot{q}_{2 \max }\right) .
\end{array}\right.
$$

\section{Endurance performance.}

Because the energy is mainly consumed in driving the joint motors, two measures can be adopted to extend the robot lifetime: reducing the energy consumption of the motors and minimizing the inertia of the legs. Decreasing the linkage dimensions is an effective measure to reduce the inertia. It can also reduce the mechanism space, which is advantageous for the spatial layout. Therefore, the objective functions of the endurance capacity can be expressed by the following equations:

$$
\left\{\begin{array}{l}
\min f_{5}(x)=\min \left(l_{1}+h_{2}+h_{3}\right), \\
\min f_{6}(x)=\min (E) .
\end{array} .\right.
$$

By combining with the constraints and the objective functions, a multi-objective optimization model of the proposed three-segment leg is established:

$$
\left\{\begin{array}{l}
\min F(x)=\min \left[f_{1}(x), f_{2}(x), f_{3}(x),\right. \\
\left.\quad f_{4}(x), f_{5}(x), f_{6}(x)\right] ; \\
x=\left(l_{1}, h_{2}, h_{3}\right) .
\end{array}\right.
$$

\section{Multi-objective optimization model}

In the multi-objective optimization model, there is more than one objective to be minimized or maximized. These objective functions are competing or conflicting with each other such that a small change in an independent variable can result in an increase in one of the objectives and a decrease in another. In this study, the multi-objective genetic algorithm was employed to find the optimal tradeoff solution between the conflicting objectives.

\subsection{Gamultiobj algorithm}

Based on the improved NSGA-II algorithm, the multi-objective genetic algorithm [24](gamultiobj) works on a population using a set of operators. The initial population is generated randomly by default, and the next generation of the population is derived from the non-dominated rank, which assigned to each individual using the relative fitness, and a distance measure of the individuals in the current generation.

\subsection{Optimal selection based on the maximizing deviation method}

To select the optimal dimension of the leg from the Pareto optimal solutions, the weight of each objective function is determined based on a combination weighting method [25] that integrates the subjective weight information and the objective weight information. It can sufficiently utilize objective evaluation information to meet the requirements of the decision-maker and can also be easily performed on a computer. The detailed flowchart and realization steps are given subsequently.

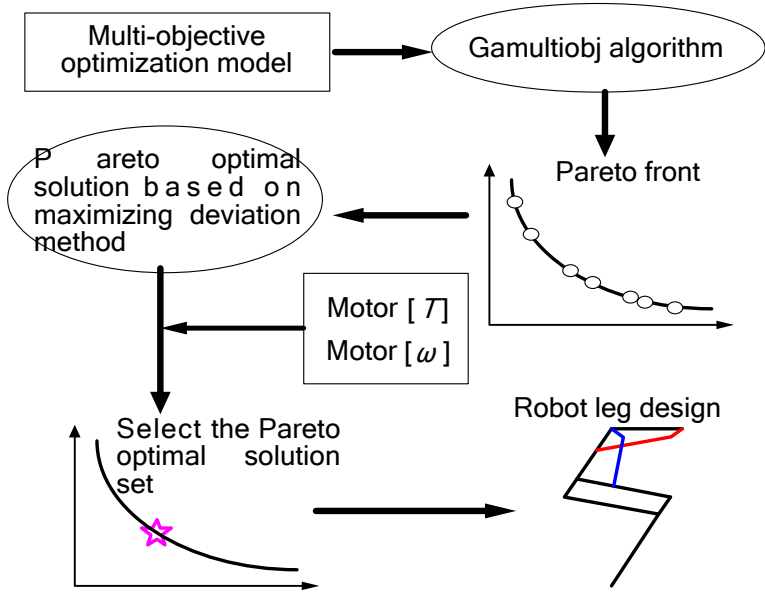

Fig. 3 Diagrammatic flow chart of Pareto selection

1. The normalized decision-making matrix $R=\left(r_{i j}\right)_{n \times m}$ is determined by transforming the Pareto op- 


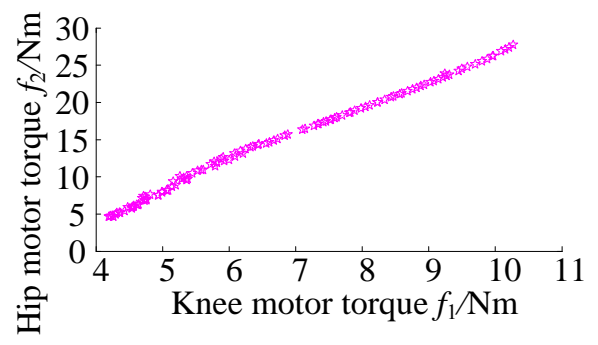

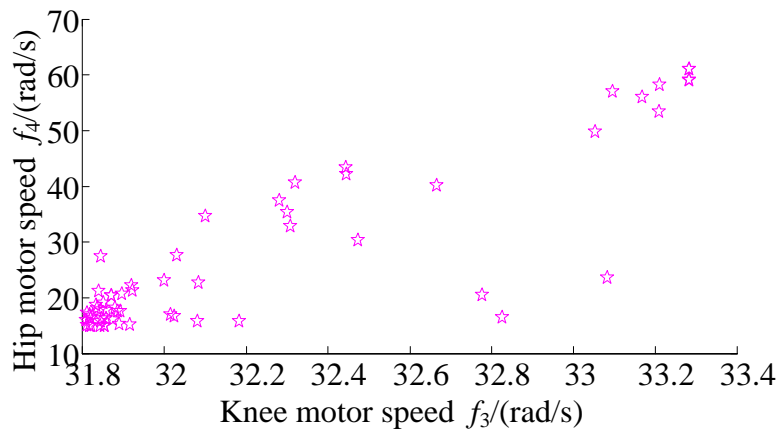

b
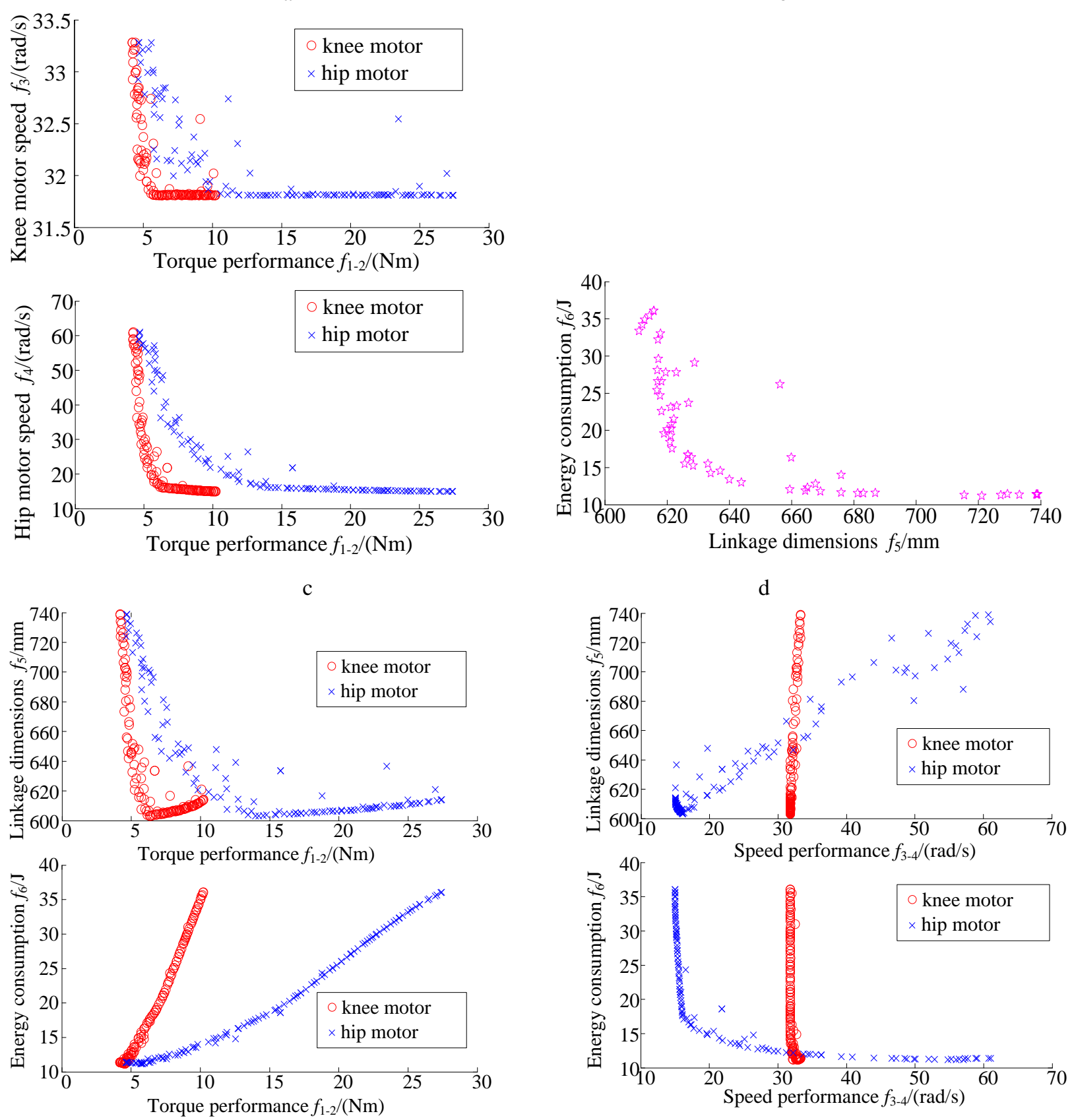

e

f

Fig. 4 Competition between each target: a - torques; $\mathrm{b}$ - speeds; $\mathrm{c}$ - speed vs torque; $\mathrm{d}$ - energy consumption vs dimensions; e - energy consumption vs torque; $\mathrm{f}$ - energy consumption vs speed

timal solution set into a decision matrix and performing normalization processing, where $m$ and $n$ are the numbers of attributes and decisions, respectively.
2. The subjective weight of each attribute $\omega=\left(\omega_{1}, \omega_{2}, \cdots, \omega_{m}\right)^{T}$ is determined using the analytic hierarchy process method [26] . 
3. Combined with the normalized decision matrix $R$, the objective weight of each attribute $\mu=\left(\mu_{1}, \mu_{2}, \cdots, \mu_{m}\right)^{T}$ is determined using the information entropy method.

4. The subjective and objective weights of each attribute are combined by using the maximizing deviation method to obtain the comprehensive weights to enable correct estimations and decisions.

\subsection{Optimization and analysis}

The gamultiobj algorithm parameters are provided in the following Table 2 .

Table 2

Parameter settings of gamultiobj algorithm

\begin{tabular}{|c|c|}
\hline Parameter & Value \\
\hline Population quantity $N$ & 400 \\
\hline Evolution generations $G_{\max }$ & 600 \\
\hline Optimal front individual coefficient $P_{F}$ & 0.3 \\
\hline Crossover probability $P_{C}$ & 0.8 \\
\hline Mutation probability $P_{m}$ & 0.5 \\
\hline Crossover distribution index $\eta_{C}$ & 20 \\
\hline Mutation distribution index $\eta_{m}$ & 20 \\
\hline
\end{tabular}

The competitive relationship among the objective functions is obtained by drawing the Pareto optimal solution set as shown in Fig. 4.

Figs. 4, a and b show the competition relationships of the torque and speed performances, respectively, between the hip and knee motor. When the dimensions are changed, the torque performance and the speed performance have the same change tendency, either increasing or decreasing at the same time. The torque range and speed range of the hip motor are greater than those of the knee motor. This means that the torque and speed sensitivity of the hip motor are higher. It can clearly be observed from Fig. 4, c that the speed will decrease upon increasing the torque in either of the motors. Therefore, we should make a good tradeoff between the speed and torque to meet the requirements of the robot velocity and load at the same time. Fig. 4, d shows that the total energy consumption decreases upon increasing the linkage dimensions, but the larger the linkage dimensions, the greater the robot's mass and the less stable the robot's motion.

As we can clearly see from Fig. 4, e and Fig. 4, f, the linkage dimensions' decrease and the total energy consumption increases with the torque increase for both the hip and knee motor, while the linkage dimensions' increase and the total energy consumption decreases with the speed increase for the motor. The speed of the knee motor does not change with the linkage dimensions or total energy consumption changes and maintains a steady value of approximately $31.8-33.4 \mathrm{rad} / \mathrm{s}$, while the speed of the hip motor is more sensitive and competitive. Set the Pareto optimal solution obtained by the gamultiobj algorithm as the decision matrix and the objective functions as the attributes, and then use the maximizing deviation method to obtain the comprehensive weights shown in Table 3.

Using the linear weighting method [25], the weighted sum is obtained. The optimal sets sorted by the weighted sum are shown in Table 4.

Considering the constraints of the allowable torque and allowable speed of the motor, select a set of solutions with the maximum weighted sum as the optimal solution to guarantee the optimal comprehensive properties of the three-segment leg. Then, the final linkage dimensions are rounded to facilitate machinery processing, to obtain final values of $l_{1}=75 \mathrm{~mm}, l_{2}=170 \mathrm{~mm}, l_{3}=22 \mathrm{~mm}$, $l_{4}=130 \mathrm{~mm}, h_{1}=110 \mathrm{~mm}, h_{2}=32.5 \mathrm{~mm}, h_{3}=105.5 \mathrm{~mm}$, and $h_{4}=20 \mathrm{~mm}$.

Table 3

Comprehensive weights

\begin{tabular}{|c|c|c|}
\hline Attribute & \multicolumn{2}{|c|}{ Weight } \\
\hline \multirow{2}{*}{$\begin{array}{c}\text { Torque per- } \\
\text { formance }\end{array}$} & Hip motor & 0.23278 \\
\cline { 2 - 3 } & Knee motor & 0.22593 \\
\hline \multirow{2}{*}{$\begin{array}{c}\text { Speed perfor- } \\
\text { mance }\end{array}$} & Hip motor & 0.13199 \\
\cline { 2 - 3 } & Knee motor & 0.14898 \\
\hline $\begin{array}{c}\text { Endurance } \\
\text { performance }\end{array}$ & Linkage dimensions & 0.11766 \\
\cline { 2 - 3 } & Energy consumption & 0.14263 \\
\hline
\end{tabular}

Table 4

Optimal solutions of the parameters

\begin{tabular}{|c|c|c|c|c|c|c|c|c|c|}
\hline \multirow{2}{*}{$\begin{array}{c}\text { Weighted } \\
\text { sum }\end{array}$} & \multicolumn{3}{|c|}{ Optimal solutions/mm } & \multicolumn{6}{c|}{ Performance parameters } \\
\cline { 2 - 10 } & $l_{1}$ & $h_{2}$ & \multirow{2}{*}{$h_{3}$} & \multicolumn{2}{c|}{$\begin{array}{c}\text { Torque perfor- } \\
\text { mance, } \mathrm{Nm}\end{array}$} & \multicolumn{2}{c|}{$\begin{array}{c}\text { Speed perfor- } \\
\text { mance, rad/s }\end{array}$} & \multicolumn{2}{c|}{$\begin{array}{c}\text { Endurance perfor- } \\
\text { mance }\end{array}$} \\
\cline { 5 - 11 } & & & & $f_{1}$ & $f_{2}$ & $f_{3}$ & $f_{4}$ & $f_{5}, \mathrm{~mm}$ & $f_{6}, \mathrm{~J}$ \\
\hline 0.841 & 75.19 & 32.48 & 105.71 & 4.577 & 6.861 & 32.578 & 32.325 & 665.173 & 12.262 \\
\hline 0.831 & 65.73 & 31.20 & 105.20 & 4.692 & 7.200 & 32.109 & 30.957 & 646.091 & 12.345 \\
\hline 0.791 & 69.87 & 34.80 & 108.73 & 4.736 & 7.080 & 32.322 & 33.240 & 661.033 & 12.071 \\
\hline
\end{tabular}

\section{Experimental analysis}

The developed robot leg terramechanics testbed consists of three main components, namely, the real testbed, control software, and simulator. Fig. 5 depicts an overview of the whole system.

\subsection{Equipment}

The developed soil testbed setup, depicted in Fig. 5, consists of a steel soil bin of $40 \mathrm{~cm}$ width, $120 \mathrm{~cm}$ length and $30 \mathrm{~cm}$ height, filled up to $20 \mathrm{~cm}$ with soil. This soil bin is large enough to avoid side wall and bottom effects in the system level test runs for robot legs within the 
dimensions of the actual robot leg prototypes. Besides performing the soft soil experiment, a wooden board can be installed on the soil bin to conduct a walking experiment on a hard surface.

In this study, a high power density DC brushless motor (Maxon EC 45, 200-W) was selected as the hip motor and knee motor. The output torque can reach $15 \mathrm{Nm}$ with a GP 42C gear box. The EPOS2 50/5 motor driver is selected to communicate with an industrial computer using the CANopen protocol. To precisely measure the speeds and torques generated by the motor, a JN-DNJ 50 digital torque and rotational speed sensor is installed between the robot leg and the motor.

The leg movement trajectories are generated using control software written in C\#.
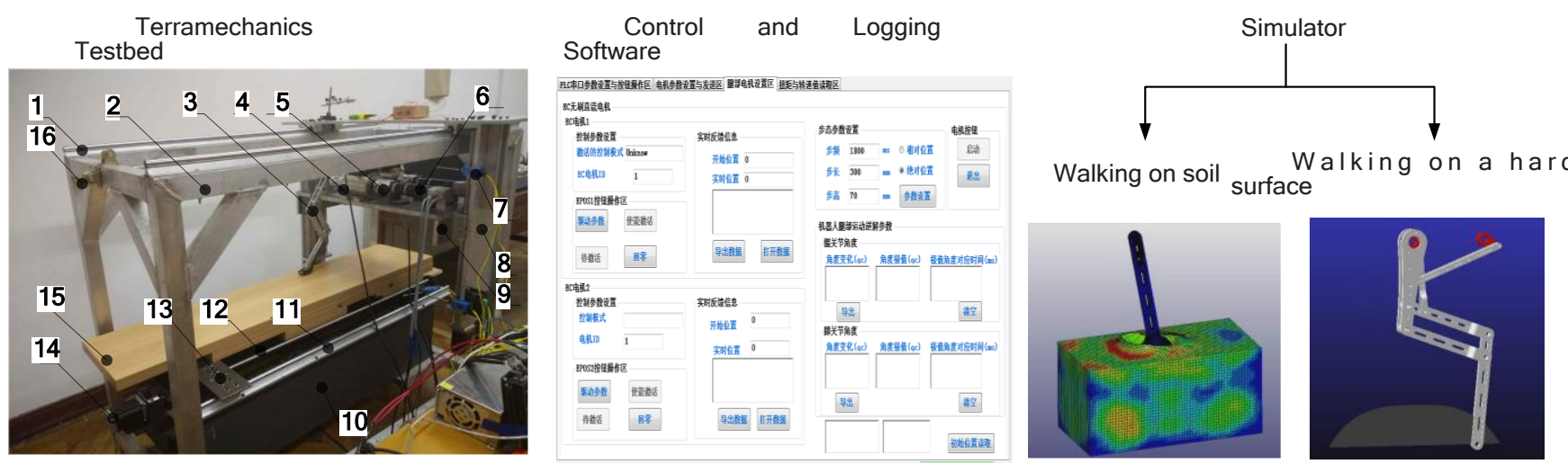

Fig. 5 Robot leg terramechanics testbed architecture overview: 1 - guideway I; 2 - frame; 3 - three-segment leg; 4 - digital torque and rotational speed sensor; 5 - coupling; 6 - DC brushless motor; 7 - limit switch 2; 8 - screw slide module; 9 - supporting worktable; 10 - soil bin; 11 - guideway II; 12 - ball screw; 13 - scraper; 14 - stepper motor; 15 - wood; 16 - fixed pulley

\subsection{Walking experiment of prototype}

In addition, multiple lightening treatment is adopted using finite element analysis software to decrease the leg's mass and rotary inertia under the premise of guaranteeing the safety of the leg strength. Considering the fact that a tendon-bone co-location architecture not only provides compliance in the leg but can also reduce the bone stresses caused by bending on the structures, we attach an elastic tendon to the ankle to reduce the pulse stress on the leg. Finally, the prototype robot leg is built as shown in Fig. 6.

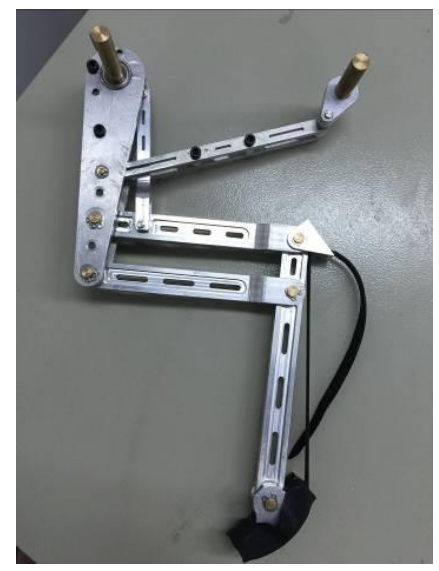

Fig. 6 Prototype of proposed leg

To conduct walking experiments, we first generate the foot trajectories using the low contact impact gait planning method according to the motion parameters in Table 1. Then, we calculate the angle of the hip motor and knee motor by inverse kinematics. To coordinate the hip motor and the knee motor, the Interpolated Position Mode in EPOS2 50/5 is used, where the trajectory is calculated by the industrial computer and passed on to the driver's interpolated position buffer as a set of points. The driver then reads the points from the buffer and performs linear or cubic interpolation between them.

No-load walking experiments are carried out on the hard surface and on the soil to verify the maximum walking speed of the robot. It is found through experiments that the minimum gait period may reach $0.6 \mathrm{~s}$. In other words, the maximum walking speed can reach $1 \mathrm{~m} / \mathrm{s}$, which is 2.2 times the leg length per second. The image of the robot is captured by a portable camera every $0.06 \mathrm{~s}$, as shown in Fig. 7.

As clearly shown in Fig. 7, the foot trajectories are consistent with the design. In the walking experiment on the hard surface, the real-time speed and torque data output by the motor are acquired by the JN-DNJ 50 and transmitted through the USB protocol to the industrial computer. The data in a gait period is recorded and then compared with the ADAMS simulation results and Matlab calculation results, respectively, as shown in Fig. 8.

From Fig. 8, it is evident that the robot leg is in the swing phase from $0.3 \mathrm{~s}$ to $0.6 \mathrm{~s}$. At this point, the torques of both the simulation results and calculation results are nearly zero. However, the actual measured results generally maintain small values due to the existence of friction and installation errors. From 0 to $0.3 \mathrm{~s}$, the leg is in the static phase. In this case, the torques of both the hip motor and knee motor increase dramatically because of the ground reaction force.

Because the payload and the inertia moment of hip motor is greater than the case of knee motor, and the joint friction between the hinges isn't taken into account in the simulation and calculation, the discrepancy between the experimental and computational results is larger in the 

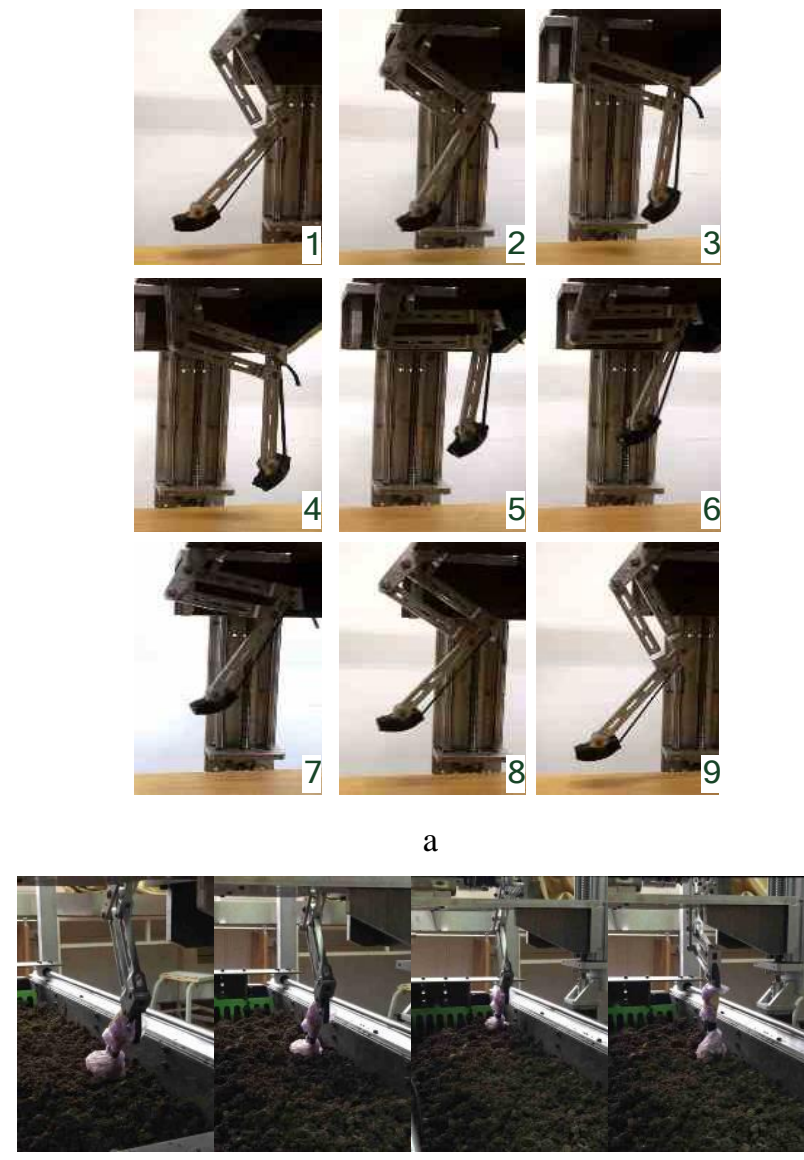

b

Fig. 7 Walking experiment of prototype: a - on the hard surface; $b$ - on the soil

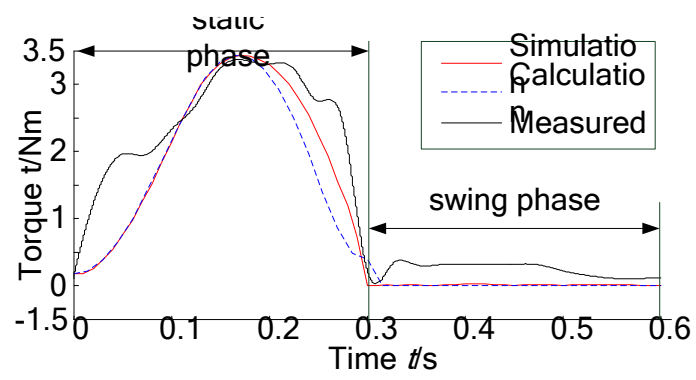

a

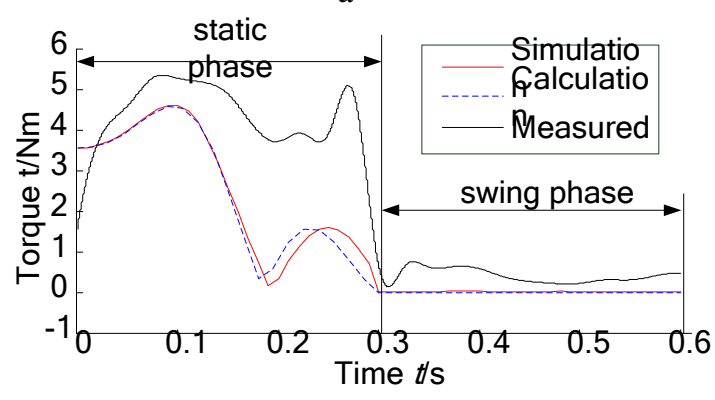

b

Fig. 8 Comparison chart of the motor torque: a - knee motor; $\mathrm{b}$ - hip motor

case of the hip motor in comparison to the case of knee motor. In addition, the difference between the assumed constant ground reaction force in the simulation and calculation and the actual constantly changing force causes the increasing error for hip motor, which is more likely to be affected by the payload. Although there are a few small differences among the measurement, simulation and calculation results, the overall trends of the curves are the same. These differences between the calculation results and the measured results maintain nearly fixed values. These errors have little interference on the optimization results of the proposed leg.

\section{Conclusions}

1. A novel electric drive robot leg based on the double four-bar mechanism is proposed. Both the hip motor and knee motor are set up near the shoulder to minimize the inertia of the leg, and the crank rocker mechanism is used to transform the continuous rotation of the motors to the back and forth motion of the leg. While making the prototype, multiple lightening treatment is adopted under the premise of guaranteeing the safety of the leg strength, and an elastic tendon is attached. These improvement measures make the realization of the high-speed movement possible with a light-weight leg.

2. The link dimensions have a significant impact on the robot performance, such as the peak torque, peak angular velocity, and total energy consumption. The mathematical relationships are set up by carrying out kinematic and dynamic analysis. The maximizing deviation method is adopted to integrate the subjective weight information and the objective weight information, which helps decision-makers select the optimal solution.

3. The results of the theoretical calculation and simulation are well coincident with those of experiments. The maximum walking speed can reach $1 \mathrm{~m} / \mathrm{s}$, which is 2.2 times the leg length per second.

\section{Acknowledgements}

This research was supported by the National Natural Science Foundation of China (51405239) and the Fundamental Research Funds for the Central Universities (KY2201761).

\section{References}

1. Hyun, D.J.; Seok, S.; Lee, J.; et al. 2014. High speed trot-running: Implementation of a hierarchical controller using proprioceptive impedance control on the MIT Cheetah. The International Journal of Robotics Research 33(11): 1417-1445. http://dx.doi.org/10.1177/0278364914532150.

2. Maes, L.D.; Herbin, M.; Hackert, R.; et al. 2008. Steady locomotion in dogs: temporal and associated spatial coordination patterns and the effect of speed. Journal of Experimental Biology 211(1): 138-149. http://dx.doi.org/10.1242/jeb.008243.

3. Ananthanarayanan, A.; Azadi, M.; Kim, S. 2012. Towards a bio-inspired leg design for high-speed running. Bioinspiration \& biomimetics 7(4): 4-12. http://dx.doi.org/10.1088/1748-3182/7/4/046005.

4. Walter, R.M.; Carrier, D.R. 2007. Ground forces applied by galloping dogs. Journal of Experimental Biology 210(2): 208-216. http://dx.doi.org/10.1242/jeb.02645.

5. Liu, J.; Zhao, X.G.; Tan, M. 2006. Legged robots: a review. Robot 28(1): 81-88. 
http://dx.doi.org/10.13973/j.cnki.robot.2006.01.017.

6. Seok, S.; Wang, A.; Chuah, M.Y.; et al. 2013. Design principles for highly efficient quadrupeds and implementation on the MIT cheetah robot.2013 IEEE International Conference on Robotics and Automation (ICRA). IEEE: 3307-3312. http://dx.doi.org/10.1109/ICRA.2013.6631038.

7. Farve, N.N. 2012. Design of a low-mass high-torque brushless motor for application in quadruped robotics. Massachusetts Institute of Technology. http://hdl.handle.net/1721.1/75658.

8. Estremera, J.; Waldron, K.J. 2008. Thrust control, stabilization and energetics of a quadruped running robot. The International Journal of Robotics Research 27(10): 1135-1151.

http://dx.doi.org/10.1177/0278364908097063.

9. Sprowitz, A.; Tuleu, A.; Vespignani, M.; et al. 2013. Towards dynamic trot gait locomotion: Design, control, and experiments with Cheetah-cub, a compliant quadruped robot. The International Journal of Robotics Research 32(8): 932-950. http://dx.doi.org/10.1177/0278364913489205.

10. Hutter, M.; Gehring, C.; Bloesch, M.; et al. 2012. StarlETH: A compliant quadrupedal robot for fast, efficient, and versatile locomotion.15th International Conference on Climbing and Walking Robot-CLAWAR 2012.

http://dx.doi.org/10.1142/9789814415958_0062.

11. Lewis, M.A.; Bunting, M.R.; Salemi, B.; et al. 2011. Toward ultra high speed locomotors: design and test of a cheetah robot hind limb.Robotics and Automation (ICRA), 2011 IEEE International Conference on. IEEE: 1990-1996. http://dx.doi.org/10.1109/ICRA.2011.5979812.

12. Raibert, M.; Blankespoor, K.; Nelson, G.; et al. 2008 Bigdog, the rough-terrain quadruped robot. Proceedings of the 17th World Congress. Proceedings Seoul, Korea, 10822-10825. http://dx.doi.org/10.3182/20080706-5-KR-1001.01833.

13. Sapaty, P. 2015. Military Robotics: Latest Trends and Spatial Grasp Solutions. International Journal of Advanced Research in Artificial Intelligence 4(4): 9-18.

14. Pratt, J.; Pratt, G. 1998. Intuitive control of a planar bipedal walking robot.1998 IEEE International Conference on Robotics and Automation. IEEE: 2014-2021. http://dx.doi.org/10.1109/ROBOT.1998.680611.

15. Nichol, J.G.; Singh, S.P.; Waldron, K.J.; et al. 2004 System design of a quadrupedal galloping machine. The International Journal of Robotics Research 23(1011): 1013-1027. http://dx.doi.org/10.1177/0278364904047391.

16. Kaneko, K.; Kajita, S.; Kanehiro, F.; et al. 2002. Design of advanced leg module for humanoid robotics project of METI.2002 IEEE International Conference on Robotics and Automation. IEEE: 38-45. http://dx.doi.org/10.1109/ROBOT.2002.1013336.

17. Lambrecht, B.G.; Horchler, A.D.; Quinn, R.D. 2005. A small, insect-inspired robot that runs and jumps. Proceedings of the 2005 IEEE International Conference on Robotics and Automation. IEEE, 1240-1245. http://dx.doi.org/10.1109/ROBOT.2005.1570285.

18. Park, J.; Kim, K.; Kim, S. 2014. Design of a catinspired robotic leg for fast running. Advanced Robotics 28(23): 1587-1598. http://dx.doi.org/10.1080/01691864.2014.968617.

19. Chai, H.; Meng, J.; Rong, X.W.; et al. 2014. Design and implementation of SCalf, an advanced hydraulic quadruped robot. Robot 36(4): 385-391. http://dx.doi.org/10.13973/j.cnki.robot.2014.0385.

20. Blickhan, R.; Seyfarth, A.; Geyer, H.; et al. 2007. Intelligence by mechanics. Philosophical Transactions of the Royal Society of London A: Mathematical, Physical and Engineering Sciences 365(1850): 199220.

http://dx.doi.org/10.1098/rsta.2006.1911.

21. Fischer, M. S.; Blickhan, R. 2006. The tri-segmented limbs of therian mammals: kinematics, dynamics, and self-stabilization - a review. Journal of Experimental Zoology Part A: Comparative Experimental Biology 305(11): 935-952. http://dx.doi.org/10.1002/jez.a.333.

22. Wang, L.P.; Wang, J.Z.; Wang, S.K.; et al. 2013. Strategy of foot trajectory generation for hydraulic quadruped robots gait planning. Journal of Mechanical Engineering 49(1): 39-44. http://dx.doi.org/10.3901/JME.2013.01.039.

23. Li, N.; Wang, M.H.; Ma, S.G.; et al. 2012. Mechanism-parameters design method of an amphibious transformable robot based on multi-objective genetic algorithm. Journal of Mechanical Engineering 48(17): 10-20.

http://dx.doi.org/10.3901/JME.2012.17.010.

24. Funke, L.W.; Schmiedeler, J.P.; Zhao, K. 2015. Design of planar multi-degree-of-freedom morphing mechanisms. Journal of Mechanisms and Robotics 7(1): 11007. http://dx.doi.org/10.1115/1.4029289.

25. Zhou, H.; Sun, J.; Yeow, B. S.; et al. 2016. Multiobjective parameter optimization design of a magnetically actuated intravitreal injection device. In Advanced Motion Control (AMC), 2016 IEEE 14th International Workshop on IEEE. IEEE: 430-435. http://dx.doi.org/10.1109/AMC.2016.7496388.

26. Sen, D.K.; Datta, S.; Patel, S.K.; et al. 2015. Multicriteria decision making towards selection of industrial robot: exploration of PROMETHEE II method. Benchmarking: An International Journal 22(3): 465487. http://dx.doi.org/10.1108/BIJ-05-2014-0046.

Yongnian Zhang, Xinsheng Wang, Yuhong Xin, Yang Wu, Min Kang, Xiaochan Wang

\section{QUADRUPED ROBOT LEG OPTIMIZATION BASED ON A MULTI-OBJECTIVE GENETIC ALGORITHM}

S u m m a r y

To improve the speed of a quadruped robot, a novel electric-drive robot leg based on a double four-bar mechanism is proposed. This scheme avoids the need for constant alternation between the acceleration and deceleration of the motors and may thus achieve a higher rotation speed. However, the dimensional parameters of the double four-bar mechanism have a significant impact on the dynamic performance of the robot. To determine the optimal dimensions, a kinematic and dynamic analysis of the pro- 
posed robot leg is carried out, and the functional relations between the joint motors' peak torque, peak angular velocity and total energy consumption in a single gait cycle and the dimensional parameters are determined. In this way, a multi-objective optimization model for the leg design is constructed, and the gamultiobj algorithm is applied to obtain the Pareto optimal solution set of the optimization model. By determining the weight of each objective function based on a combination weighting method, the optimal dimensions of the leg are obtained from the Pareto optimal solution. Finally, a virtual prototype of the robot leg is built via ADAMS to conduct a walking simulation, and the physical prototype is installed on a self-developed test bench of one-legged movement to conduct a no-load walking experiment. The results of the theoretical calculations and simulation are well coincident with those of the tests, which verify the correctness and validity of the proposed algorithm.

Keywords: Double four-bar mechanism; Gamultiobj algorithm; Pareto optimization; robot leg.

Received January 08, 2017 Accepted December 07, 2017 